Horizons philosophiques

\title{
De la nature au social : espace et historicité du jugement de goût
}

\section{Michel Peterson}

Volume 4, numéro 1, automne 1993

Théories esthétiques

URI : https://id.erudit.org/iderudit/800932ar

DOI : https://doi.org/10.7202/800932ar

Aller au sommaire du numéro

Éditeur(s)

Collège Édouard-Montpetit

ISSN

1181-9227 (imprimé)

1920-2954 (numérique)

Découvrir la revue

Citer cet article

Peterson, M. (1993). De la nature au social : espace et historicité du jugement de goût. Horizons philosophiques, 4(1), 31-52. https://doi.org/10.7202/800932ar d'utilisation que vous pouvez consulter en ligne.

https://apropos.erudit.org/fr/usagers/politique-dutilisation/ 


\title{
De la nature au social : Espace et historicité du jugement de goût
}

\author{
À l'esthétique de tirer au clairle passage de telle forme de \\ vie à telle autre.
}

Daniel Charles'

L'imbrication de l'esthétique et du politique ne fait aucun doute. Elle pose toutefois un certain nombre de problèmes dont le plus fondamental est sans doute celui de la polémique du sujet créateur avec la société à laquelle il appartient. Qu'il accepte, conteste ou relativise les formes du vivre-ensemble, l'artiste est toujours quelque peu en porte-à-faux et trouve souvent dans son isolement, réel ou fantasmé, une «raison» de s'exprimer. Cherchant à éclairer le rôle joué par la solitude dans le travail des peintres, Malraux note en effet :

Précurseurs parfois comblés de l'art, Rembrandt et Goya n'avaient pas conçu leur vocation comme celle de la solitude. C'estla solitude qui révèleà Goya sa vocation. Au XIXe siècle, une solitude particulière, féconde et contemptrice, devient liée à la vocation méme de l'artist $\theta^{2}$.

Ce propos illustre de façon très précise l'appel à la solitude de l'artiste ou plutôt cette sorte d'obligation perverse, qui se présente à l'artiste, de se retirer. Une parole et une voix semblent lui faire signe, lui susurrer à l'oreille : «Retire-toi du monde car toujours davantage ton oeuvre s'enfoncera dans la lumière de la nuit. Tu disparaîtras!» Comment Van Gogh entendit-il cette voix? L'expression que se prête déjà Goya dans un autoportrait de jeunesse réalisé en 1774 nous l'apprend:

II nous montre un homme, aux yeux profonds, au front haut et à la figure assez large; c'est un visage à la fois résolu et pensif. [...] Dans cette oeuvre, le chapeau caractéristique, les sourcils touffus, les favoris épais, la large cravate, donnent l'image d'un homme qui, sans être coquet, suit cependant la mode. Cela lui donne

1. "Esthétique", «Histoire de l'esthétique», Encyclopaedia Universalis, tome 7, Paris, 1985, p. 298a.

2. André Malraux, Le Musée imaginaire, Paris, Gallimard, coll. “ldées/Arts», 1965, p. 65. 
l'air de grande dignité qui convient à un artiste universellement estimé. Mais un sentiment de solitude émane du regard, de la bouche et de l'expression même du visage. La surdité de Goya met une barrière entre lui et le monde. II ne peut franchir cet obstacle que partiellement, grâce à l'acuité de son regard qui semble ici se poser fugitivement, et presque de biais, sur le spectacle qu'il contemple ${ }^{3}$.

En se représentant lui-même comme un être solitaire ne maintenant que peu de contacts avec le monde qui l'entoure, Goya accuse la dichotomie artiste-individu/société. Il est d'ailleurs remarquable que le sentiment d'abandon se manifeste dans son regard, d'autant plus qu'il est déjà sourd au moment où il peint la première planche des Caprices. C'est donc avec un regard évanescent et oblique qu'il observe le monde. En devenant son oreille, l'oeil de Goya ${ }^{4}$ lui révèle son absence non seulement au collectif, mais aussi, et surtout, à l'art.

C'est que si l'esthétique et le politique sont intimement liés, l'art et la solitude n'existent que l'un par l'autre. À la question : "dans l'épreuve de la création, qu'en est-il de la solitude?", Maurice Blanchot propose d'ailleurs une réponse convaincante :

3. M. Lepore, Francisco Goya, adapt. fr. J.M. Charlier, Paris, Dargaud, 1971, p. 74. [C'est nous qui soulignons.]

4. On pourrait montrer de quelle manière se joue ici l'articulation de deux sens que Hegel considérait comme les deux sens métaphysiques par excellence, à savoir l'ouïe et la vue. Et pourtant... quelque chose d'autre est déjà en marche, un travail qu'on pourrait qualifier, si le terme n'était pas anachronique, de «déconstructeur" puisque la différance de la vérité et de l'art se déploie dans un espace où la production irréductible au concept importe davantage que la production comme telle d'une vérité de l'expression. Si, pour reprendre Derrida, "le tympan louche", c'est parce que l'oeuvre d'art opère une destruction radicale des rapports et des moyens d'expression fondés sur l'évidence de la présence : «Luxer, tympaniser l'autisme philosophique, cela ne s'opère jamais dans le concept et sans quelque carnage de la langue. Celle-ci alors défonce la voûte, l'unité close et volutée du palais. Elle prolifère au dehors jusqu'à n'être plus comprise. Elle n'est plus la langue." "Tympan", Marges de la philosophie, Paris, Minuit, 1972, p. vii, note 4. Comment ne pas être sensible à cette utympanisation" en jugeant $L \theta$ Rapt de Ganymède ou La Résurrection de Lazare du maître Rembrandt? Et que dire des planches des Désastres de Goya, des madrigaux de Monteverdi, du Marteau sans maîtrede Boulez-Charou des «interprétations" (des "silences") deBach, Beethoven, Ives, Krenek ou Schoenberg par Glenn Gould? 
Celui qui écrit l'oeuvre est mis à part, celui qui l'a écrite est congédié. Celui qui est congédié, en outre, ne le sait pas. Cette ignorance le préserve, le divertit en l'autorisant à persévérer. L'écrivain ne sait jamais si l'oeuvre est faite. Ce qu'il a terminé est un livre, il le recommence ou le détruit en un autre. 5

Comme le peintre, le danseur ou le sculpteur, l'écrivain disparaît totalement dans son oeuvre. L'artiste lui-même devient, par la solitude, par l'oeuvre qui le rejette en cette solitude qu'il ignore, personne. Cette expérience de la dépossession du sujet est l'expérience fondamentale de l'art. Le sujet s'efface, disparaît et s'abîme dans la matière de l'oeuvre toujours à recréer. Dans les autoportraits exécutés par Goya, où est le peintre? Goya reconnaît-il son propre visage? Si oui, pourquoi repeint-il sans cesse d'autres autoportraits? Tout se passe comme si l'artiste comprenait que l'oeuvre, comme l'analyse, est définitivement interminable.

Si la création semble dans la plupart des cas avoir lieu dans la solitude, la réception apparaît toutefois comme le moment capital de l'expérience esthétique où s'affirment le devenir-autre et le devenir-collectif de l'oeuvre. La forme du collectif et la forme du jugement se conjuguent dans la réception et font ainsi passer l'oeuvre du plan de l'individualité subjective à celui de l'universalité intersubjective. Dans la mesure où le passage de la création à la réception met en jeu le sujet dans son rapport aux formes disponibles dans le monde, il est par conséquent nécessaire de remonter au lieu où s'articulent le vivre-ensemble et le jugement de goût.

\section{Esthétique et vivre-ensemble}

Peu importe sur quelles bases historico-philosophiques et dans quels domaines de connaissance l'on considère aujourd'hui l'esthétique et le politique, c'est évidemment à Kant qu'il revient d'avoir théorisé leur rapport premier dans sa

5. Maurice Blanchot, L'Espace littéraire, Paris, Gallimard, coll. «idées», 1955, p. 10. 
dimension critique. ${ }^{6}$ Dès le $\S 6$ de la troisième Critique, la parenté du jugement de goût avec le jugement logique apparaît dans toute sa netteté puisque l'on doit présupposer pour l'un comme pour l'autre un assentiment universel. La première définition du beau vient tout juste d'être formulée (il est l'objet d'une satisfaction désintéressée) en fonction de la qualité et il faut donc maintenant adopter le point de vue de la quantité afin d'en venir à poser que le beau est ce qui plaît universellement sans concept. En bref, le jugement de goût donne lieu à un plaisir (Wohlgefallen) désintéressé convenant a priorià l'humanité. Kant précise :

Cependant cette universalité Allgemeinheit ne peut résulter de concepts. II n'existe en effet pas de passage Uebergang des concepts au sentiment de plaisir ou de peine (exception faite dans les pures lois pratiques qui entraînent un intérêt, tandis que le pur jugement de goût n'est lié à rien de tel). II s'ensuit que la prétention de posséder une valeur pour tous doit être liée au jugement de goût et à la conscience d'être dégagé de tout intérêt, sans que cette prétention dépende d'une universalité fondée objectivement; en d'autres termes, la prétention à une universalité subjective doit être liée au jugement de goût. $(\S 6)^{7}$.

C'est parce que le pont conceptuel ne peut être construit que s'impose la nécessité de la prétention (Anspruch — droit, revendication, réclamation) à l'universalité subjective, laquelle contient en soi la raison d'être de la liberté. L'usage de celle-ci "exige l'adhésion des autres, <loin> de compter sur leur adhésion [...]." Contrairement à l'agréable, le jugement de goût exclut de manière radicale la simple appréciation individuelle car : «Cela reviendrait à dire : le goût n'existe pas, il n'existe pas

6. Et ce même si, comme le rappelle Louis Guillermit dans L'Élucidation critique du jugement de goût selon Kant (Paris, CNRS, 1986, p. 32, note 74), la chaîne jugement-réflexion-altérité est déjà formée chez Muratori (Delle riflessioni sopra il buon gusto nelle scienze e nell'arti, 1708), Scaliger (Poetices libri septem, 1561) et Hume (A Treatise of Human Nature, 1737).

7. Critique de la faculté de juger, Paris, Vrin, 1982, p. 56. Nous n'indiquerons désormais que le paragraphe. [C'est nous qui soulignons]. 
de jugement esthétique qui pourrait légitimement prétendre à l'assentiment de tous." (§ 7) Alors que le geste de la création engageait l'artiste dans sa propre et indivise solitude, le jugement de goût se fonde donc, dans sa forme pure, sur l'intersubjectivité et sur la possibilité de la communication. Alors que le sublime réduit les sujets au silence le plus douloureux, le beau, plaisir indépendant de tout intérêt, ouvre l'espace du vivre-ensemble. L'universalité non-conceptuelle (esthétique) génère par conséquent des quantités subjectives dont chacune constitue, selon l'expression de Kant, une valeur commune (Gemeingültigkeit).

II serait évidemment inutile de multiplier les exemples. Rappelons simplement que la communicabilité universelle s'effectue non dans le désordre de la sensibilité empirique, mais dans l'ordre d'un plaisir pur (celui de la répétition) livré dans le jugement réfléchissant. Dans un texte où il rétablit, en posant le vomi comme parergon de la troisième Critique, le quasi secret rapport unissant mimesis et oikonomia, Derrida souligne d'ailleurs que ce plaisirne peutêtre donné que "[...] selon l'ordre d'un certain socius, d'une certaine intersubjectivité réfléchissante ${ }^{8} "$. Toute la question est évidemment de savoir quel type d'économie encourage une telle intersubjectivité. La réponse est, à la lettre, anéconomique. Le plaisir amputé du concept et de la jouissance est le plaisir (Lust) le plus pur qui soit : «De l'homme libre : capable de productivité pure, c'est-àdire non-échangeable. [...] Ni comme valeur d'usage, ni comme valeur d'échange ${ }^{9}$." Nous voici donc parvenus au-delà et en deçà de la solitude. En d'autres termes, nous voici arrivés à l'ère de «l'économie pure» : qualitativement désintéressé, le plaisir esthétique répond à un désir qui ne constitue en fait que (constitue surtout : en cela il est animé par l'intérêt le plus puissant qui soit: il cherche à transformer les représentations en réalité) la relation des représentations à leur réalisation. II ne se

8. «Economimesis», Sylviane Agacinski et als., Mimesis des articulations, Paris, Aubier-Flammarion, 1975, p. 66.

9. Ibid., p. 66-67. 
rapporte par conséquent d'aucune façon au désir intéressé défini dans la Critique de la raison pratique comme cause de l'existence de ce qu'il représente, cause de la réalité de ses objets. L'esthétique peut produire (faire apparaître, faire connaître et faire exister) du politique, la mimesis produire un rapport entre des productions, entre des productions de productions, entre des libertés. Or cette non-imitation n'est rendue possible que sur la base d'une identification de l'humain au divin :

D'une liberté à une autre. Communicabilité des jugements purs du goût, échange (universel, infini, sans limite) entre les sujets aux mains libres dans l'exercice ou l'appréciation du bel art, tout cela suppose un commerce entre l'artiste divin et l'artiste humain. Et ce commerce est bien une mimesis au sens strict, scène, masque, identification à l'autre sur la scène, et non l'imitation d'un objet par sa copie. La "vraie" mimesis; entre deux sujets producteurs et non entre deux choses produites. Impliquée par toute la troisième Critique, bien que le thème explicite, encore moins le mot, n'y apparaisse jamais, une telle mimesis requiert la condamnation de l'imitation, toujours qualifiée de servile ${ }^{10}$.

Toujours et d'entrée de jeu conditionnelle, c'est-à-dire morale, la liberté (que ce soit celle du sujet dans son rapport spéculaire à l'autre ou celle du jeu de nos facultés de connaître) procède, comme condition de la loi morale, d'une mimesis non pas proto-reproductible, mais plutôt anti-reproductive. Peu importe alors que l'insistance de Kant sur le "bon goût» dans l'esthétique accentue la rupture entre la politique et la raison en plus de la provoquer entre la politique et la morale, car même si la relation entre l'ordre moral et l'ordre esthétique se trouve maintenue à l'aide de la règle analogique (l'intérêt moral intervenant toujours dans le désintéressement esthétique), il n'en demeure pas moins que le partage entre la morale et la politique repose, comme tel, sur l'esthétique ${ }^{11}$.Qu'il pense à une commu-

10. Ibid., p. 66-67.

11. David Cook and Arthur Kroker, The Postmodem Scene. Excremental Culture and Hyper-Aesthetics, Montreal, New World Perspectives, 1987, p. 161-162. 
nauté des hommes ou à une communauté des esprits, à des individus concrets ou à des êtres idéels, Kant reste formel quant au caractère social du goût sans pour autant tomber dans la mystique ou l'idéalisme d'une éthique communicationnelle qui guiderait vers une utopique communion intersubjective.

Le jugement de goût permet toutefois de fonder le politique, de prétendre à l'universalité, non seulement parce que les conditions subjectives de la faculté de juger sont identiques chez tous les hommes, mais parce qu'il traduit en outre un choix : "Le goût, écrit Kant dans ses Réflexions, est le pouvoir de choisir de façon universellement valable." Louis Guillermit commente ce passage de la façon suivante : «[...] s'il s'élève audessus du simple plaisir qui s'attache à la sensation, c'est que, tout en restant dans la sphère du sensible, il tente de s'arracher à la seule valeur "privée" de la subjectivité pour l'accorder à celle d'autrui ${ }^{12}$." Si le choix ne s'opère pas sur la base d'un intérêt pour le contenu, c'est que l'art ne constitue, dans la perspective kantienne, qu'une technique (tekhnè) formée par un ensemble de règles objectives. Voilà pourquoi la communauté dans laquelle le jugement de goût nous invite à entrer présuppose (et mène vers) une conception du Beau comme symbole d'une moralité ( $\$ 59$ : le beau est le symbole du bien moral et cette relation, précise Kant, est naturelle à chacun et chacun l'attend des autres comme un devoir) renvoyant à la Nature. D'une liberté à l'autre, d'une idée de la raison spéculative à l'autre, serons-nous alors forcés de subsumer la multitude des intentionnalités distinctes sous une loi morale qui détermine la volonté, de ramener l'hétérogène à l'homogène?

La difficulté de cette question indique en tout cas que le rapport établi par Kant entre l'esthétique et le politique s'avère plus complexe que nous l'avions jusqu'à présent supposé. Les conséquences entraînées par la réponse, qu'elle soit positive ou négative, s'avèrent évidemment si lourdes dans le processus de formation des groupes humains qu'il nous faut, sans

12. L'élucidation..., p. 31. Le texte de Kant est tiré par Guillermit des corrections qu'il apporte à la deuxième édition de la Critique de la Raison pure. 
entrer dans des détails dont l'analyse dépasserait largement le cadre de nos réflexions, considérer quelques instants le lien qui unit la nature et la liberté d'une part, et la difficulté du passage du singulier à l'universel d'autre part. Si l'esthétique et le politique renoncent l'un comme l'autre à l'argumentation rationnelle en résistant à la force subsumante des concepts objectifs (d'où le fait qu'ils voilent en quelque sorte tous deux la violence qui donne accès à l'histoire), leur approche respective du geste qui consiste à créer (par donation, legs, attribution, représentation, exclusion, etc.) une forme d'existence collective diffère radicalement. Alors que la forme du collectif légitime en quelque sorte la nature religieuse de sa naissance même si, comme le souligne avec raison Luc Ferry, l'apparition de l'esthétique correspond à un certain effacement du divin ${ }^{13}$, la forme du jugement offre malgré tout une résistance active à tout acte de fondation.

Tandis que Hegel rejette le Beau naturel parce que la beauté fait apparaître l'ldée dans l'oeuvre d'art et que la nature constitue une réalisation de l'Esprit, Kant s'emploie au contraire à faire de la nature le lieu où s'expérimente l'altérité. Samuel Weber constate à ce sujet: "In this text [la troisième Critique], what nature signifies is not anything reassuringly familiar but rather something impenetrably opaque. Nature names the place where judgment encounters its "other", a heterogeneity and diversity which resist subsumption under general concepts $^{14}$." L'inquiétante étrangeté du moment de la rencontre du jugement avec l'autre se manifeste lorsque la nature entre dans

13. Homo Aestheticus. L'invention du goûta à l'âge démocratique, Paris, Grasset, 1990, p. 45. Cet effacement ou ce retrait du divin met d'ailleurs l'esthétique, comme le précise Ferry, face à de nouvelles interrogations : l'esthétique doit upenser le «sens commun» suscité par l'objet beau, ou, si l'on veut, l'accord des "sensibilités" (du moins, bien sâr, lorsqu'ila alieu), autrement que de façon théologique (monadologique). En d'autres termes, c'est maintenant à une certaine représentation de l'intersubjectivité qu'on doit recourir pour comprendre un tel accord."

14. * The Foundering of Aesthetics: Thoughts on the Current State of Comparative Literature", The Comparative Perspective on Literature. Approaches to Theory and Practice, Clayton Koelb and Susan Noakes (eds), Ithaca and London, Cornell University Press, 1988, p. 64. 
le domaine du particulier et n'appartient plus, comme dans la Critique de la raison pure, au domaine du général. L'introduction de la troisième Critique est, sur ce point, on ne peut plus claire : "La finalité de la nature est [...] un concept particulier a priori, qui a son origine uniquement dans la faculté de juger réfléchissante." L'opacité de la nature tient - non moins que celle des groupes humains - au fait qu'elle relève en définitive d'une diversité impossible à systématiser puisque le sujet se trouve toujours, dans un jugement réfléchissant, face au particulier. C'est en quelque sorte ce que laisse entendre Kant au moment d'énoncer le principe de la faculté de juger, lequel, «[...] en ce qui concerne la forme des choses de la nature sous des lois empiriques en général, est la finalité de la nature en sa diversité, ce qui signifie que par ce concept on se représente la nature comme si [als ob] un entendement contenait le principe de l'unité de la diversité de ses lois empiriques." L'intégration de la diversité dans l'unité, c'est-à-dire l'accord d'une chose avec la constitution des choses (accord qui ne se conclut que si la fin de l'objet est réalisée) demeure, du moins en principe, impossible à concrétiser au niveau de la raison. Le jugement esthétique n'est pas synthétique, n'est pas un jugement de connaissance. Tout au plus nous permet-il de nous représenter le concept de la nature comme si nous pouvions coordonner les données de l'expérience.

II faut donc qu'intervienne le jugement réfléchissant lequel reflète davantage son propre désir que le jugement d'un objet extérieur à lui - pour que s'accomplisse la finalité de la nature : «[...] on ne peut faire usage de ce concept [la finalité de la nature en tant que concept particulier a priori] que pour réfléchir sur la nature au point de vue de la liaison des phénomènes en celle-ci, liaison donnée d'après des lois empiriques" (Introduction). Or le propre du jugement réfléchissant est de ne jamais devenir déterminatif puisqu'il consiste en un mouvement du particulier vers l'universel. Et comme le rappelle Weber, c'est ce «mouvement vers» qui oriente les efforts de Kant dans la troisième Critique dans la mesure où le jugement occupe une position intermédiaire entrel'universel et le particulier: «Aesthetic 
judgment is occupied with "finding" the universal, which is not given in the particular case. To found aesthetics, in this sense, is to found a certain effort to find the universal. Aesthetical judgment, therefore, finds, but what it finds can never be founded, if by this we mean justified, legitimated, demonstrated, or falsified ${ }^{15}$." Comment, en effet, l'imagination libre qui réfléchit un objet singulier pourrait-elle s'harmoniser dans le goût avec l'entendement si l'universel était donné dans le variable? Dès lors que l'accord subjectif des facultés fonde un sens commun ( $\$ 20,21$ et 22), la communicabilité du sentiment sans concept devient possible.

Le sens commun esthétique ouvre ainsil'espace du vivreensemble. Lorsqu'il travaille à calculer l'intérêt empirique que prennent les hommes au beau, Kant écrit :

\begin{abstract}
Le beau n'intéresse empiriquement que dans la société; et si l'on admet que la tendance < Triebs à la société est naturelleàl'homme, mais quel'aptitude et le penchant pour la société, c'est-à-dire la sociabilité< <ie Geselligkeit>, sont nécessaires à l'homme en tant que créature destinée à vivre en société, et constituent une propriété appartenant àl'humanité, on ne peut manquer de considérer également le goút comme une faculté de juger ce qui permet de communiquer même son sentiment à tout autre et par conséquent comme un moyen de réaliser ce qu'exige l'inclinaison naturelle de chacun. (§ 41)
\end{abstract}

Le sensis communis aestheticus se trouve toutefois aussitôt rejeté. Kant refuse de lui accorder l'importance qu'il mérite non parce qu'il reste sourd, loin s'en faut, à la matérialité et à l'empiricité des sociétés (son anthropologie, ses réflexions sur la nature des races humaines et sur l'histoire universelle, etc., suffisent amplement à prouver le contraire), mais parce que l'objectif de la faculté de juger consiste ici à éviter le passage très équivoque (zweideutiger Übergang) de l'agréable au bon

15. Ibid., p. 66. C'est dans l'Analytique du sublime que sera pour ainsi dire résolu le problème du passage du singulier à l'universel. 
révélé par l'empirique afin d'établir la relation avec le jugement de goût a priori. Mais ce projet n'empêche nullement, une fois acceptée la contrainte qu'impose la culture, de lier la finalité de la nature à la finalité morale. La méthodologie du goût devient alors évidente : "[...] la véritable propédeutique pour fonder le goût est le développement des ldées morales et la culture du sentiment moral, puisque ce n'est que si l'on accorde la sensibilité avec celui-ci que le goût authentique peut prendre une forme déterminée et invariable» ( $\$ 60$, «Appendice» à l'Analytique du sublime). Un tel accord implique bien sûr un questionnement préalable de la nature du lien qui unit le sujet individuel au sujet collectif. À ce stade-ci, il faut citer tout au long le passage dans lequel Kant fournit, avant de passer à la critique de la faculté de juger téléologique, les bases d'une étude rigoureuse des beaux-arts :

La propédeutique pour tous les beaux-arts, lorsqu'il s'agit du suprême degré de leur perfection, ne semble pas consister dans des préceptes, mais dans la culture des facultés de l'âme grâce à ces connaissances préliminaires, que l'on nomme humaniora, sans doute parce que humanité signifie d'une part le sentiment universel de sympathie $<$ Teilnehmungsgefühb, d'autre part la faculté de pouvoir se communiquer d'une manière intime et universelle < das Vermögen, sich innigst und allgemein mitteilen zu können>; ces qualités réunies constituent la sociabilité convenant à l'espèce humaine, grâce à laquelle elle se distingue de l'animalité bornée. $(\S 60)$

L'analyse des rapports des sujets au groupe passe alors, à travers l'histoire de la "dialectique» liberté/contrainte, au plan du droit :

Le siècle aussi bien que les peuples, en lesquels la tendance active à une sociabilité légale <gesetzlicher Geselligkeits, qui constitue un peuple en un corps commun durable, luttait avec les grandes difficultés qui s'attachent au grave problème d'unir la liberté (et par conséquent la légalité) avec la contrainte (plutôt par respect et soumission au devoir que par crainte), - ce siècle et ces peuples devaient tout d'abord inventer l'art de la communication 
réciproque des Idées <die Kunst der wechselseitigen Mitteilung der ldeen entre les classes les plus cultivées et les plus incultes, l'adaptation du développement et du raffinement des premières à la simplicité naturelle et à l'originalité des secondes et ainsi découvrir entre la culture supérieure et la simple nature le moyen-terme, qui constitue également pour le goût, en tant que sens commun de l'homme, l'exacte mesure, qui ne peut être donnée par des règles générales (Ibid.).

Anticipant (ouvrant...) la modernité esthético-politique, Kant conclut enfin en anticipant l'inévitable rupture qui surgira, au sein même des peuples, entre la force (Kraft) et la justesse (Richtigkeit) de la nature libre et la légalité de la culture.

Reprenons brièvement à partir du $\S 41$ et suivons le trajet qui conduit Kant du postulat selon lequel la société est la condition sine qua non du beau considéré sur le mode empirique (sans concept) jusqu'au moment où il propose de lier nature et culture par une contrainte légale et légitime (la première Déclaration des droits de l'homme et du citoyen vient tout juste d'être votée par l'Assemblée nationale constituante). Si nous voulions montrer les réelles dimensions du rapport unissant le beau à la socialité, il faudrait évidemment revenir à l'Esthétique transcendantale de la première Critique pour ensuite traverser la Métaphysique des moeurs et ses Fondements. Contentonsnous ici de souligner l'importance capitale du goût dans la genèse en quelque sorte empirique de la communication. La faculté de juger est à la communicabilité universelle ce que la socialité est à l'humanité. On peut alors poser la question du criticisme : quelle est la condition a priori (ce que Goethe appellera l'Urphänomenon, le phénomène premier) de la société? Réponse : le beau, c'est-à-dire cela qui plaît universellement sans concept. Voilà pourquoi l'humanité est définie par l'universalité du sentiment de sympathie et du 
pouvoir de se communiquer de façon intime. L'opposition privé/ public devient, dans ce contexte, difficile à maintenir selon ses préceptes bourgeois ${ }^{16}$. Irons-nous jusqu'à prétendre que l'esthétique donne forme au politique dont «la priorité théorique et ontologique" en tant qu' 'institution du sens" semble incontestable ${ }^{17}$ ? Une telle extrapolation n'étant bien sûr possible que sur la base d'un examen de la syntaxe profonde du collectif, nous devons d'abord comprendre comment se nouent la destinée supra-sensible (destinée entrevue grâce à l'art) et la destinée morale du sujet.

\section{Relationnisme et historicisme}

Formulée sous la forme d'une contrainte nécessaire (cf. $\S 60$ ), la culture se rapporte au devoir-être par le biais de la finalité. En marge des esthétiques des $X I X^{\ominus}$ et $X X^{\ominus}$ siècles, sans doute les analyses de Wittgenstein sont-elles parmi celles qui, à la suite de Kant, rétablissent avec le plus de rigueur la chaîne esthétique-éthique, même si elles refusent le romantisme et l'organicisme qui lui succèdent. Aussi éloignée que soit la philosophie wittgensteinienne du langage du criticisme kantien (l'écart entre les formes a priori de la sensibilité et l'espace logique qui, contenant les faits, forme le monde), quelques points communs entre le relationnisme sociologique de la première et le subjectivisme contemplatif du second méritent d'être soulignés.

Entre le renversement par Baumgarten et la reprise par Bergson de la notion d'intuition, les modalités des expériences artistiques se sont multipliées à tel point que l'esthétique ne sera plus jamais en mesure de prononcer des jugements quant à ce qui est beau ou non. Si Wittgenstein se démarque entièrement de Kant en ce qui concerne le caractère subjectif du

16. L'ampleur de sa réflexion sur le rapport entre le beau et l'universalité exonère évidemment Kant des accusations d'ascétisme et d'égoïsme esthétique. À ce sujet, Silvestro Marcucci, Intelleto $\theta$ intellettualismo» nell'estetica di Kant, Ravenna, Longo Editore, 1976, p. 144 et ss.

17. André Akoun, "Société», Encyclopaedia Universalis, tome 16, Paris, 1985, p. 1047 b. 
jugement de goût, il partage avec lui l'idée qu'il est impossible (mais pour des raisons différentes) de constituer l'esthétique en domaine d'étude systématique. C'est que l'esthétique cherche, tout comme l'éthique, à construire une sémantique du monde tel qu'il se donne comme ensemble des faits (de la totalité des faits) et non des choses ${ }^{18}$. D'où l'impossibilité radicale où se trouve placé l'esthéticien de fonder sa discipline en théorie. Dans son commentaire des Leçons sur l'esthétique, Jacques Bouveresse insiste d'ailleurs sur la difficulté de définir le domaine de l'esthétique :

Cette incompréhension résulte, pour l'essentiel, du fait qu'on représente l'esthétique avant tout comme la théorie (ou la philosophie) du Beau ou du plaisir esthétique. On commet [...] une erreur analogue lorsqu'on considèrel'éthique comme une interrogation sur l'essence du Bien ou du Devoir; et Wittgenstein remarque, dans les Cours de 193033 : "Pratiquement tout ce que je dis sur «beau» s'applique d'une manière légèrement différente à «bon»". Si vous cherchez explicitement ce qui fait qu'un objet est beau ou une action bonne, vous ne pourrez rien trouver, et aucune des choses intéressantes et importantes que vous pourriez trouver par ailleurs sur eux ne sera la chose ${ }^{19}$.

L'art moderne ayant entrepris de se déployer selon un matérialisme formel dont les bases ont été jetées par Kant luimême ${ }^{20}$, la subjectivité et l'objectivité du jugement esthétique deviennent l'une comme l'autre inaptes à décrire l'expérience tant du sujet que de l'objet esthétique. Pour Wittgenstein, seule demeure la culture, laquelle renvoie non seulement à l'acte (au moment positif) de fondation des groupes humains, mais à la communauté de vie des individus. Sa contribution à l'esthétique concerne davantage le langage et les formes de vie (c'est-àdire le sens) qu'une prétendue Science du Beau à partir de

18. Tractatus logico-philosophicus, art. 1.1 et 1.11, trad. fr. Pierre Klossowski, Paris, Gallimard, 1961, p. 43.

19. Wittgenstein : la rime et la raison. Science, éthique et esthétique, Paris, Minuit, 1973, p. 155.

20. $A$ ce sujet, nous nous permettons de renvoyer à notre article : "Le trident de Neptune", in Trois, vol. 3, no. 3, printemps-été 1988, p. 159-163. 
laquelle il serait possible d'identifier un certain nombre de propriétés communes à l'ensemble des oeuvres. C'est pourquoi l'esthétique est ainsi, avec le domaine des recherches dites conceptuelles, le domaine de recherche qui intéresse le plus Wittgenstein.

Le sens de l'identification esthétique/éthique surgit certes à travers les perspectives que nous ouvrent les valeurs circulant dans la culture. II faut toutefois régresser en deçà même du vivre-ensemble si nous voulons saisir l'importance de l'esthétique pour la cohérence du politique. Selon Wittgenstein, l'éthique et l'esthétique forment en fait un seul et même domaine de connaissance (ils appartiennent au même ensemble axiologique) dans la mesure où, à l'instar de la logique, ils ne constituent pas des discours sur le monde mais bien la condition (Bedingung) même du monde. Dans la mesure où sont assimilés le monde et la vie, où la vie estle monde ${ }^{21}$, l'on comprend que le jugement esthétique ne puisse se pratiquer, contrairement à l'acte de création, qu'à l'intérieur d'une communauté vivante.

Une fois reconstituée la chaîne esthétique-éthique-politique à son niveau le plus élémentaire, il nous faut considérer le jugement esthétique tel que le conçoit Wittgenstein : "When we make an aesthetic judgement about a thing, we do not just gape at it and say: "Oh! How marvellous!» We distinguish between a person who knows what he is talking about and a person who doesn't." L'exemple qui suit est remarquable: "If a person is to admire English poetry, he must know English ${ }^{22}$." Le jugement esthétique se caractérise donc par la capacité qu'il nous donne à la fois d'apprécier le beau et de réaliser une distinction entre les individus qui savent et ceux qui ne savent pas. En d'autres termes, le jugement esthétique wittgensteinien ressemble à s'y méprendre au jugement synthétique au sens où le définissait

21. Notebooks. 1914-1916, Chicago, Chicago University Press, 1979, p. 77-77e.

22. Lectures and Conversations on Aesthetics, Psychology, and Religious Belief, Berkeley, University of California Press, 1966, p. 6. James Taylor commente ainsi la définition de Wittgenstein : "He must react in a consistent way over a long period. Must know all sorts of things" (p. 6, note 1). 
Kant. On ne peut en effet statuer sur le savoir d'un individu donné que si nous faisons appel à l'expérience. Il faut que Pierre dise : "Ceci est beau» ou : "Cette personne est belle» pour que nous puissions légitimement affirmer : «Pierre sait» - ce qui suppose (comme lorsqu'on dit : "Pierre est debout») que l'on ne découvre la vérité ou la fausseté du jugement esthétique de Pierre qu'a posteriori. Plus encore : si l'on admet avec Wittgenstein que l'éthique et l'esthétique sont un seul et même type de rapport au monde et à la vie, il faut également admettre que la proposition : "Pierre sait» implique une appréciation de Pierre. Apprécier Pierre consiste à déterminer si les attitudes qu'il développe à partir de son savoir entrent ou non en contradiction avec le système de valeurs que nous laisse en héritage la culture à laquelle nous appartenons. D'où l'importance accordée au juge dans le jugement (éthico-)esthétique, lequel ne s'applique d'ailleurs pas uniquement aux beaux-arts. Prolongeant la pensée de Wittgenstein selon laquelle le jugement dans le domaine des beaux-arts est un développement, Rush Rees ajoute: "In what we call the arts there is developed what we call a «judge" - i.e. one who has judgement. This does not mean just someone who admires or does not admire. We have an entirely new element ${ }^{3}$." Or cet élément nouveau était déjà d'une certaine manière présent dans la troisième Critique. La réponse esthétique ne relève pas d'un subjectivisme psychologique, lequel ferait dépendre les valeurs esthétiques de l'expérience strictement individuelle et rendrait alors difficile l'établissement de règles validant ou invalidant les jugements de goût. Le juge a pu devenir un acteur essentiel des beaux-arts parce qu'il a su faire de sa judication un acte dont le résultat, c'est-à-dire le jugement comme tel, permet d'obtenir un partage fondé essentiellement sur le savoir et non sur le pouvoir ou la connaissance.

23. Ibid., p. 6, note 3. 
Le problème de l'absence de fondements objectifs des jugements esthétiques reste cependant entièrement irrésolu. À la troisième Critique qui en arrive à poser une faculté de juger dialectique et raisonnante - «[...] c'est-à-dire que ses jugements doivent prétendre à l'universalité et cela même a priori' (§56) - dont l'antinomie du goût constitue l'expression parfaite (§57), Wittgenstein «répond» en proposant un jugement esthétique qu'on peut qualifier de jugement de relation a posteriori. Celui-ci renvoie à l'ensemble de la culture, laquelle correspond évidemment à un jeu de langage donné. Le jugement est donc une activité fort complexe qui ne peut être liée à la connaissance que si elle s'intègre dans un bassin de valeurs et de jeux de langages : "The judgement, précise Rees, is a gesture accompanying a vast structure of actions not expressed by one judgemen ${ }^{4}$." Cette structure, c'est-à-dire la culture, motive le choix de valeurs que nous portons sur la base du jugement esthétique ou de ce que Wittgenstein appelle l'appréciation. D'où, à partir de prémisses logico-linguistiques, la mise en place d'une logique de relations impliquant une relation entre le sujet et l'attribut. Dire d'un objet ou d'un être qu'il est beau implique alors, dans le cas qui nous intéresse, que l'environnement culturel soit décisif pour nos motivations axiologicoesthétiques ${ }^{25}$. En relativisant le goût, en lui enlevant son caractère absolu, l'on peut alors savoir (déterminer) si Pierre sait ou ne sait pas. Le jugement esthétique relève par conséquent, selon Wittgenstein, du procès historique : "To describe their use [les mots] or to describe what you mean by a cultured taste, you have to describe a culture. What we now call a cultured taste perhaps does not exist in the Middle Ages. An entirely different game is played in different ages ${ }^{26}$." Le fait que la définition historique du goût dépende, avec son existence, d'une description préalable de la culture explique qu'il faille se fier non à la

24. Ibid., p. 11, note 2.

25. A ce sujet, voir Stefan Morawski, Inquiries into the Fundamentals of Aesthetics, Cambridge-London, MIT Press, 1974, p. 31.

26. Lectures..., p. 8. 
forme des mots ou des oeuvres, mais bien aux activités au sein desquelles ils sont produits.

L'esthétique de Wittgenstein est donc effectivement, comme on a pu l'affirmer, une "esthétique appliquée" ou un "criticisme pratique" au sens où il constitue un domaine d'application de règles ${ }^{27}$. Ce domaine est précisément celui qui autorise le juge à signifier des jugements dont la légitimité provient à la fois de l'Idée de nature et du jugement de goût, lequel repose, si l'on se fie à Kant, "sur une simple sensation de l'animation réciproque de l'imagination dans sa liberté et de l'entendement dans sa légalité». (§ 35) La grave responsabilité que doit assumer le juge est ainsi fonction de sa capacité à prononcer un jugement qui prend acte - afin de la réduire en en ordonnant les conséquences pratiques - de l'écart entre la connaissance et la liberté :

Le juge supplée à l'absence d'un tribunal universel ou d'un jugement dernier devant lequel le régime de la connaissance et celui de la liberté pourraient être, sinon réconciliés, ils ne le seront jamais, mais du moins mis en perspective, ordonnés, finalisés selon leur différence. Cette suppléance est cependant autorisée par l'ldée d'une nature, au sens kantien. Nature est le nom que porte l'objet de I'ldée de finalité objective, et celle-ci est elle-même exigée par le jugement réfléchissant lorsqu'il cherche à rendre raison des existences singulières que la légalité du monde déterminé "mécaniquement" n'explique pas (KRV: 334). Mais si, inversement, l'activité de discerner, la Genauigkeit, l'attention donnée aux différends, qui est à l'oeuvre dans la critique, peut se charger de cette suppléance en invoquant la finalité objective d'une nature, c'est qu'elle est ellemême ('activité critique) un moyen mis en oeuvre par la nature pour préparer sa fin dernière $\left(K U K:\right.$ § 84) ${ }^{28}$.

27. Ces définitions sont de Francis J. Coleman, «A Critical Examination of Wittgenstein's Aesthetic", The Philosophy of Wittgenstein, vol. 14. Aesthetics, Ethics and Religion, John V. Canfield ed., New York-London, Garland Publishing, 1986, p. 25.

28. Jean-François Lyotard, Le Différend, Paris, Minuit, 1983, p. 195. Les abréviations $K R V$ et KUK renvoient respectivement à la Kritik der reinen Vernunft et à la Kritik der Urteilskraft. 
Lorsque le juge porte un jugement réfléchissant sur des existences singulières, il met du même coup en lumière les différends (les motifs d'hérétogénéité) qui façonnent pour ainsi dire le collectif et orientent les voies du vivre-ensemble. Mais la distinction wittgensteinienne entre celui qui sait et celui qui ne sait pas ne devient dans ce contexte appréciable que si le juge connaît les règles lui permettant de raffiner son jugement. Le juge peut alors se prononcer sur la question de savoir si tel objet ou telle personne est non pas beau ou belle, mais correcte ou incorrecte. II importe toutefois par-dessus tout que les règles orientant le jugement esthétique incluent à la fois les techniques artistiques (par exemple le contrepoint en musique, la prosodie en poésie, etc.) et l'ensemble des éléments et des valeurs qui façonnent la culture d'une époque donnée ${ }^{29}$.

Or les règles qui assurent le fonctionnement du criticisme pratique de Wittgenstein obéissent aux deux tendances dont témoignent les réflexions sur l'esthétique, à savoir le relationnisme et l'instrumentalisme (c'est-à-dire le naturalisme linguistique). Ces deux approches, qui renvoient à des théories différentes de la valeur artistique, appellent toujours, comme on l'a maintes fois remarqué, des réponses esthétiques adressées en fonction d'une situation concrète et d'une pratique linguistique précise. Elles permettent en outre de bien cerner la position sociologique de Wittgenstein à propos de la valeur artistique. "The general relationist's logic, souligne en effet Stefan Morawski, is clear : he will describe objective qualities as the "reasons" which justify his statement : the cautious instrumentalist, however, will describe only such "motives" as are shared by the social group to which he belongs (he will, nonetheless, usually go on to cite causes for his sociological preferences andaestheticnorms) $)^{30}$." Tandis que le relationnisme s'oppose tout autant à la théorie kantienne de la contemplation et du désintéressement (le détachement relevant de toute

29. Nous corroborons ici le point de vue de Coleman, "A Critical...» p. 27 et ss. 30. Op. cit., p. 31. 
façon davantage de l'attitude esthétique que de la réelle expérience esthétique) qu'à l'attitude non-pratique observée par certains post-kantiens (cette attitude combinant le désintérêt pour l'expérience vitale de l'immédiateté, au désintérêt pour le passé et le futur), l'instrumentalisme cherche à légitimer de manière ethnocentrique ses jugements en les rapportant à son groupe social. S'il est difficile de dire lequel des deux points de vue prédomine (en fait, ils se réfractent), on peut sans doute essayer de voir s'il n'est pas possible, tout en acceptant que tel jeu de langage commande un type donné de communication, de déplacer l'alternative pour comprendre l'articulation entre l'esthétique et le politique en se plaçant sur un terrain transhistorique.

Dans cette perspective, l'approche historiciste de Morawski serait sans doute l'une des plus adéquates. La question qui oriente les enquêtes de ce dernier dans le domaine de l'esthétique est la suivante: des valeurs esthétiques qui apparaissent et puissent traverser plusieurs périodes et lieux géographiques sont-elles concevables et si oui, selon quels critères? Sur le plan méthodologique, il faut donc commencer par chercher les valeurs récurrentes de l'art dans l'histoire. La bonne marche de ce travail implique quatre postulats : $1^{\circ}$ l'évidence de la continuité de l'histoire; $2^{\circ}$ la possibilité de trouver les bases de valeurs dans la nature; $3^{\circ}$ la continuité, l'évidence et la permanence des propriétés artistiques (ces caractéristiques renvoyant aux traits "relativement objectifs" qui furent perçus, au moment de leur création, comme des valeurs artistiques); $4^{\circ}$ un doute historique. Nous ne pouvons accepter les trois premiers postulats que si nous insistons sur le dernier. II serait en effet naiff de croire à la continuité de l'histoire sans adopter simultanément une attitude critique devant la permanence absolue de quelque valeur que ce soit. Ceci admis, la classification des théories de la valeur artistique établie par Morawski devient plus claire. Le subjectivisme psychologique, l'objectivisme ontologique, le sociologisme et le relationnisme trouvent tous, chacun selon des modalités différentes, leur fondement dans un rapport à l'histoire, laquelle constitue le lieu de la distribution 
concrète des valeurs et, par voie de conséquence, celui où se construit la grammaire transculturelle du collectif.

Une interrogation pertinente sur l'esthétique qui éviterait le problème de l'axiologie se condamnerait donc à être radicalement coupée de la culture. Du même coup, elle s'empêcherait de penser la forme dans son rapport actif et positif au vivre-ensemble. Le créateur et les récepteurs se trouveraient paralysés devant cette expérience - une oeuvre - dont la richesse provient du fait qu'elle engage simultanément le singulier et l'universel à l'intérieur du procès éthique. Exclus de l'incontournable rapport entre l'esthétique et le politique, comment pourraient-ils répondre à cette prescription existentielle que Witold Gombrowicz aurait désigné comme l'impératif moral de la forme? Comment les sujets pourraient-ils communiquer si la Forme ne leur permettait pas de se constituer au contact de l'autre?

Fondamentalement esthétique, l'être humain se trouve activement confronté aux formes du monde. Traversé par l'autre, son essence et son existence sont historiques et sociales - même lorsque, comme le créateur, il se croit seul et qu'il éprouve la nostalgie d'un monde à venir où il serait au centre d'une communauté impossible. L'être humain, rappelle en effet Gomborowicz, est un carrefour où se croisent, dans l'instabilité la plus pure, toutes les possibilités humaines:

L'homme soumis à l'interhumain est comme une épave sur une mer houleuse, il monte, il descend, il plonge dans la furie des eaux, il glisse doucement le long des vagues lumineuses, enivré de rimes et de rythmes vertigineux, il s'égare dans des directions imprévues; à travers la Forme, pénétré par les hommes jusqu'à la moelle, le voici plus puissant que soi et à soi inconnu, des chemins insoupçonnés s'ouvrent d'eux-mêmes, et parfois il ne sait plus du tout ce qui lui arrive. Il est fonction des tensions qui surgissent, des situations instables suscitées au hasard par les hommes, entre les hommes, et qui se trouvent être la résultante de poussées diverses. Cette création interhumaine, inconnue et insaisissable, détermine ses possibilités ${ }^{31}$.

31. Testament, entretiens avec Dominique de Roux, 1968 et 1977, p. 83. 
Par le biais de son esthétique éthico-formelle, Gombrowicz illustre le fait incontournable que la solitude constitue une forme du vivre-ensemble même si l'essence de l'art est de dévoiler, comme le soutenait son père spirituel Stanislaw Ignacy Witkiewicz, le Mystère de l'Existence en cherchant l'adéquation entre l'unité du moi de l'artiste et l'unité de la construction formelle de l'oeuvre. Plongé dans l'infinie diversité des hommes et transformé sans cesse par les aléas du monde et de l'histoire, l'artiste ('Unité ou l'Existence Particulière) se meut dans un espace où les temporalités se superposent et où les faits se multiplient par eux-mêmes jusqu'à constituer une culture en puissance. L'artiste ne ressent par conséquent sa solitude comme un état tragique - ou «naturel» - et positif que lorsqu'il sait pour ainsi dire matériellement et historiquement qu'il n'est pas l'unique porteur de l'oeuvre, laquelle semble pourtant exclusivement sienne. C'est pourquoi, de même que l'esthétique ne saurait constituer une théorie du Beau, l'art ne saurait répondre à quelque définition que ce soit :

L'art puise son concept dans la constellation de moments
qui se transforment historiquement et se ferme ainsi à la
définition. Son essence n'est pas déductible de son origine
à la façon de la première strate d'un sol sur laquelle chaque
couche suivante s'érige et s'écroule aussitôt qu'elle est
ébranlée $\theta^{32}$.

Le problème de l'origine de l'oeuvre d'art ne peut trouver sa "solution" que dans le rapport entre l'esthétique et le politique. C'estlà le seul assentimentuniversel quipuisse être présupposé. Comme quoi les formes de l'histoire trouvent leur force en dehors de l'objectivité, dans l'oeuvre, c'est-à-dire en ce moment où des sujets vivent universellement leur solitude en commun.

Michel Peterson Université de Varsovie

32. Theodor Adorno, Théorie esthétique, trad. fr. Marc Jimenez, Paris, Klincksieck, 1982, p. 10. (La première phrase, curieusement traduite par Jimenez, se lit dans l'original comme suit: “ Kunsthat ihren Begriffin dergeschichtlich sich verändernden Konstellation von Momenten; er sperrt sich der Definition.") 